\title{
CLASSIFICATION OF THE PERIODIC MONODROMIES OF HYPERELLIPTIC FAMILIES
}

\author{
MIZUHO ISHIZAKA
}

\begin{abstract}
We classify the periodic monodromies which are realized as the monodromies of hyperelliptic families.
\end{abstract}

\section{Introduction}

Let $\phi: S \longrightarrow \Delta$ be a proper surjective holomorphic map from a complex surface $S$ (i.e., a complex analytic manifold of dimension two) to a small disk $\Delta:=\{t \in \mathbf{C}|| t \mid<\epsilon\}$ such that $\phi^{-1}(t)$ is a nonsingular complex analytic curve of genus $g \geq 2$ for any $t \in \Delta^{*}:=\Delta \backslash\{0\}$. We call $(\phi, S, \Delta)$ a degeneration of curves. If all $\phi^{-1}(t)$ for $t \in \Delta^{*}$ are hyperelliptic curves, we call $(\phi, S, \Delta)$ a hyperelliptic family. Note that a hyperelliptic family $(\phi, S, \Delta)$ is bimeromorphic to a double covering $\psi_{0}: S_{0} \longrightarrow W_{0}:=\mathbf{P}^{1} \times \Delta$ branched along a divisor $B_{0}$ of $W_{0}$ (cf. [Ho1]). Two degenerations $(\phi, S, \Delta)$ and $\left(\phi^{\prime}, S^{\prime}, \Delta^{\prime}\right)$ are said to be topologically equivalent if there exist orientationpreserving homeomorphisms $\psi: S \longrightarrow S^{\prime}$ and $\bar{\psi}: \Delta \longrightarrow \Delta^{\prime}$ which satisfy $\phi^{\prime} \circ \psi=\bar{\psi} \circ \phi$.

For a topological equivalence class of a degeneration, we can uniquely determine the topological monodromy (called the monodromy, for short) as a conjugacy class in the mapping class group of genus $g$ (cf. [MM1], $[\mathrm{MM} 2])$. In $[\mathrm{AI}]$, we classify all the topological equivalence classes in the case of genus three.

An orientation preserving homeomorphism $f$ of a compact real surface of genus $g$ is said to be periodic if there exists an integer $n$ such that $f^{n}$ is isotopic to the identity map. The smallest positive such integer $n$ is called the period of $f$. By Kerchhoff's theorem (cf. [Ke]), for each periodic homeomorphism $f$, there exist a Riemann surface $\Sigma_{g}$ of genus $g$ and an analytic automorphism $\bar{f}: \Sigma_{g} \rightarrow \Sigma_{g}$ isotopic to $f$ such that $\bar{f}^{n}$ is the identity. For each point $P$ on $\Sigma_{g}$, we denote by $r_{P}$ the cardinality of the orbit of $P$

Received October 30, 2001.

Revised August 29, 2002, July 31, 2003.

2000 Mathematics Subject Classification: Primary 14D06; Secondary 14H45, 14H15, 57M99, 30F99. 
under $\bar{f}$, and let $l_{P}:=n / r_{P}$. Let $\delta_{P}$ be the smallest nonnegative integer such that $(\bar{f})^{r_{P}}$ is the rotation of angle $2 \pi \delta_{P} / l_{P}$ near each point in the orbit. Denote by $s_{P}$ the smallest positive integer satisfying $\delta_{P} s_{P} \equiv 1\left(\bmod l_{P}\right)$ if $\delta_{P} \neq 0$, and set $s_{P}:=0$ when $\delta_{P}=0$. The symbol $s_{P} / l_{P}$ is called the valency of the orbit of $P$.

Note that the valencies of all but a finite number of orbits are zero. The set of the positive valencies is called the total valency of $\bar{f}$ and expressed as the formal sum $\sum s_{P} / l_{P}$ of symbols.

We define the total valency of a periodic homeomorphism $f$ as the total valency of $\bar{f}$. It is well-known that the conjugacy class of a periodic map in the mapping class group is determined by its period and total valency.

Thus a periodic monodromy $[f]$ of a degeneration is determined by its period $n$ and the total valency that is expressed as $n_{1} / l_{1}+n_{2} / l_{2} \cdots+n_{k} / l_{k}$ for positive integers $l_{1}, \ldots, l_{k}$ and $n_{1}, \ldots, n_{k}$. This means that there exists an analytic automorphism $\bar{f}$ of $\Sigma_{g}$ of period $n$ as above such that its orbits of cardinality less than $n$ are $\left\{P_{1}^{(i)}, P_{2}^{(i)}, \ldots, P_{n / l_{i}}^{(i)}\right\}$ for $i=1, \ldots, k$. Moreover, $\bar{f}^{n / l_{i}}\left(P_{j}^{(i)}\right)=P_{j}^{(i)}$ for all $i$ and $j$, and $\bar{f}^{n / l_{i}}$ is isotopic to the rotation of angle $2 \delta_{i} \pi / l_{i}$ near $P_{j}^{(i)}$ for a positive integer $\delta_{i}$ satisfying $\delta_{i} n_{i} \equiv 1 \quad\left(\bmod l_{i}\right)$.

In this paper, we classify the periodic monodromies which are realized as the monodromies of hyperelliptic families (Corollary 1.7). Moreover, for a given periodic monodromy $[f]$, we can show that we can choose a branch locus of $\phi_{0}: S_{0} \longrightarrow \Delta$ from the list in Theorem 1.5 such that the monodromy of the nonsingular model of $S_{0}$ is $[f]$. Thus, for a given hyperelliptic family $S$ with periodic monodromy, we can calculate the Horikawa index (cf. [AA, $\S 4]$ ) from the period and the total valency of the monodromy of $S$.

Acknowledgements. The author wishes to express his special thanks to Professors Tadashi Ashikaga, Kazuhiro Konno, Tatsuya Arakawa, Nariya Kawazumi and Takayuki Morifuji for useful advice. He also thanks Professors Tadao Oda, Masanori Ishida, Takeshi Kajiwara and Shigeru Takamura for encouragement.

\section{\$1. Classification of periodic monodromies}

Let $(\phi, S, \Delta)$ be a hyperelliptic family of genus $g$. A complex surface $S$ is said to be normally minimal if the singularities of the reduced scheme of the special fiber are ordinary double points and any $(-1)$-curve in the special fiber intersects the other components at at least three points. In this paper, we assume that any degenerations of curves are normally minimal.

We first review Horikawa's canonical resolution of double coverings (cf. [Ho2]). It is well-known that $S$ is bimeromorphic to a double covering 
$\psi_{0}: S_{0} \longrightarrow W_{0}:=\mathbf{P}^{1} \times \Delta$ branched along a divisor $B_{0}$ of $W_{0}$ (cf. [Ho1]). More precisely, $S_{0}$ is a hypersurface in the total space of the line bundle $F_{0}$ over $W_{0}$ such that $F_{0}^{\otimes 2}$ is isomorphic to the line bundle $\left[B_{0}\right]$ associated to $B_{0}$. Let $\pi_{0}$ be the second projection of $W_{0}$ and $\Gamma_{t}$ the fiber of $\pi_{0}$ at a point $t \in \Delta$. Let $\phi_{0}$ be the composite $\pi_{0} \circ \psi_{0}$. We set $\widetilde{B_{0}}:=B_{0}-\Gamma_{0}$ when $\Gamma_{0}$ is a component of $B_{0}$, and $\widetilde{B}_{0}:=B_{0}$ otherwise. By the Hurwitz formula, the intersection number $B_{0} \Gamma_{t}$ is equal to $2 g+2$. Let $I_{P}\left(B_{0}, \pi_{0}^{-1}(t)\right)$ be the local intersection number of $B_{0}$ and $\Gamma_{t}$ at a point $P$. Since $S$ has at most one special fiber, we see that $t=0$ if $I_{P}\left(B_{0}, \pi_{0}^{-1}(t)\right) \geq 2$.

We denote the multiplicity of $B_{0}$ at $P$ by $m_{P}$ and denote the greatest integer not exceeding $m_{p} / 2$ by $\left[m_{P} / 2\right]$. Let $\tau_{1}: W_{1} \longrightarrow W_{0}$ be the blowingup at a point $P$ which is a singular point of $B_{0}$ or satisfies $I_{P}\left(B_{0}, \Gamma_{0}\right) \geq 2$. We set $\pi_{1}:=\pi_{0} \circ \tau_{1}: W_{1} \longrightarrow \Delta, E_{1}:=\tau_{1}^{-1}(P), B_{1}:=\tau_{1}^{*}\left(B_{0}\right)-2\left[m_{P} / 2\right] E_{1}$ and $F_{1}:=\tau^{*}\left(F_{0}\right)-\left[m_{p} / 2\right] E_{1}$. Since $F_{1}^{\otimes 2}$ is isomorphic to the line bundle $\left[B_{1}\right]$ associated to $B_{1}$ again, we can take a double covering $\psi_{1}: S_{1} \longrightarrow$ $W_{1}$ branched along $B_{1}$ and naturally define a bimeromorphic morphism $\widetilde{\tau}_{1}: S_{1} \longrightarrow S_{0}$. We set $\phi_{1}:=\pi_{1} \circ \psi_{1}$. Then $\phi_{0} \circ \widetilde{\tau_{1}}=\phi_{1}$.

Repeating this process, we obtain a sequence of blowing-ups $W_{r} \stackrel{\tau_{r}}{\longrightarrow}$ $\cdots \longrightarrow W_{1} \stackrel{\tau_{1}}{\longrightarrow} W_{0}$ which satisfies the following properties:

(i) $\left(\tau_{1} \circ \cdots \circ \tau_{r}\right)^{*}\left(\Gamma_{0}\right)$ transversally intersects the strict transform of $\widetilde{B_{0}}$.

(ii) $B_{r}$ is nonsingular.

The reduced scheme of the special fiber of $S_{r}$ is a normal crossing divisor by (i). $S_{r}$ is nonsingular by (ii).

Let $\widetilde{\tau}$ be the composite of the contractions of $(-1)$-curves such that $\widetilde{\tau}\left(S_{r}\right)$ is normally minimal. Then, we obtain the original normally minimal model $\phi: S \longrightarrow \Delta$. We call the above process Horikawa's canonical resolution. We call a point $P$ on $B_{i}$ a bad point if $B_{i}$ is singular at $P$ or $I_{P}\left(\left(\tau_{1} \circ \cdots \circ \tau_{i}\right)^{*}\left(\Gamma_{0}\right)_{\mathrm{red}}, \widetilde{B}_{i}\right) \geq 2$, where $\widetilde{B_{i}}$ is the strict transform of $\widetilde{B_{0}}$ by $\tau_{1} \circ \cdots \circ \tau_{i}$. In this paper, we always use $r$ as the length of the sequence of blowing-ups which satisfies the conditions (i) and (ii).

Conversely, choosing a component $E_{r^{\prime}}$ of $\left(\tau_{1} \circ \cdots \tau_{r}\right)^{*}\left(\Gamma_{0}\right)$ whose selfintersection number is -1 , we consider a blowing-down $\tau_{r}^{\prime}$ : $W_{r} \longrightarrow W_{r-1}^{\prime}$ which contracts $E_{r^{\prime}}$ to a point. We set $B_{r}^{\prime}:=B_{r}-E_{r^{\prime}}$ when $E_{r^{\prime}}$ is a component of $B_{r}$ and $B_{r}^{\prime}:=B_{r}$, otherwise. Let $m$ be the intersection number $E_{r^{\prime}} B_{r}^{\prime}$. Since $\left(\tau_{r}^{\prime}\right)_{*}\left(B_{r}+2[m / 2] E_{r^{\prime}}\right)$ is isomorphic to $\left(\tau_{r}^{\prime}\right)_{*}\left(F_{r}+\right.$ $\left.[m / 2] E_{r^{\prime}}\right)^{\otimes 2}$, we can take the double covering $\psi_{r}^{\prime}: S_{r-1}^{\prime} \longrightarrow W_{r-1}^{\prime}$ branched along $\left(\tau_{r}^{\prime}\right)_{*} B_{r}$ and naturally define a morphism $\widetilde{\tau_{r}^{\prime}}: S_{r} \longrightarrow S_{r-1}^{\prime}$. Repeating this process, we finally obtain a sequence of blowing-downs $W_{r} \stackrel{\tau_{r}^{\prime}}{\longrightarrow} \cdots \longrightarrow$ 
$W_{1}^{\prime} \stackrel{\tau_{1}^{\prime}}{\longrightarrow} W_{0}^{\prime}$ and the double covering $\psi_{r}^{\prime}: S_{0}^{\prime} \longrightarrow W_{0}^{\prime}=\mathbf{P}^{1} \times \Delta$ such that $S_{0}^{\prime}$ is bimeromorphic to $S_{r}$. We call this process an inverse of Horikawa's canonical resolution. Note that if the multiplicity of a component $E$ of $\left(\tau_{1} \circ\right.$ $\left.\cdots \circ \tau_{r}\right)^{*} \Gamma_{0}$ is one, we can find an inverse of Horikawa's canonical resolution such that $\left(\tau_{1}^{\prime} \circ \cdots \circ \tau_{r}^{\prime}\right)_{*} E$ is $\mathbf{P}^{1}$, i.e., we can consider that $\left(\tau_{1}^{\prime} \circ \cdots \circ \tau_{r}^{\prime}\right)_{*} E$ is $\Gamma_{0}$. We call this an inverse of Horikawa's canonical resolution associated to $E$.

If the monodromy of $(\phi, S, \Delta)$ is periodic, the configuration of the special fiber $F=n_{0} M_{0}+\sum_{i}^{l} \sum_{j=1}^{k_{i}} \alpha_{j}^{i} D_{j}^{i}$ satisfies the following conditions (cf. [MM1], [MM2]):

(i) Each $D_{j}^{i}$ is a nonsingular rational curve.

(ii) $M_{0}$ is a nonsingular curve of genus $g^{\prime}\left(0 \leq g^{\prime} \leq g\right)$ and $l \geq 3$ if $g^{\prime}$ is equal to zero.

(iii) The integer $n_{0}$ coincides with the period of the monodromy.

(iv) $M_{0} D_{1}^{i}=D_{j}^{i} D_{j+1}^{i}=1\left(j=1, \cdots, k_{i}-1\right), M_{0} D_{j}^{i}=0(j \geq 2), D_{j}^{i} D_{j^{\prime}}^{i}=$ $0\left(\left|j-j^{\prime}\right| \geq 2\right), D_{j}^{i} D_{j^{\prime}}^{i^{\prime}}=0\left(i \neq i^{\prime}\right)$.

(v) $n_{0}>\alpha_{1}^{i}>\cdots>\alpha_{k_{i}}^{i}$ for all $i$.

We call $M_{0}$ the main component of $(\phi, S, \Delta)$.

LEMMA 1.1. Let $E$ be a component of $\left(\tau_{1} \circ \cdots \circ \tau_{i}\right)^{*}\left(\Gamma_{0}\right)$. Assume that there exists a bad point $Q$ on $E$ and that $Q$ is not contained in the components of $\left(\tau_{1} \circ \cdots \circ \tau_{i}\right)^{*}\left(\Gamma_{0}\right)$ other than $E$. Let $E_{0}$ be the strict transform of $E$ by $\tau_{i+1} \circ \cdots \circ \tau_{r}$ and let $\sum_{j=1}^{k} n_{j} E_{j}$ be the maximal subdivisor of $\left(\tau_{1} \circ\right.$ $\left.\cdots \circ \tau_{r}\right)^{*}\left(\Gamma_{0}\right)$ which is contracted to $Q$ by $\tau_{i+1} \circ \cdots \circ \tau_{r}$. Let $\bar{\tau}: S_{r} \longrightarrow S^{\prime}$ be a composite of blowing-downs of some (-1)-curves, where the reduced scheme of the special fiber of $S^{\prime}$ is a normal crossing divisor. If $\bar{\tau}\left(\left(\psi_{r}^{*}\left(E_{0}\right)\right)_{\text {red }}\right)$ is a nonsingular curve, then not all $\psi_{r}^{*}\left(E_{j}\right)_{\text {red }}$ are contracted by $\bar{\tau}$.

Proof. We may only consider the case where all $\psi_{r}^{*}\left(E_{j}\right)_{\text {red }}(j=1,2$, $\ldots, k)$ are nonsingular rational curves. Let $n_{0}$ be the multiplicity of $E_{0}$ and assume that the dual graph of $\sum_{j=0}^{k} n_{j} E_{j}$ is linear, i.e., $E_{j} E_{j+1}=1$ for all $j$ $(1 \leq j \leq k-1)$ and $E_{j} E_{j^{\prime}}=0$ for $\left(j, j^{\prime}\right)$ which satisfy $\left|j-j^{\prime}\right| \geq 2$. Assume that all $\psi_{r}^{*}\left(E_{j}\right)(j=1,2, \ldots, k)$ are contracted by $\bar{\tau}$. Since $\bar{\tau}\left(\left(\psi_{r}^{*}\left(E_{0}\right)\right)_{\text {red }}\right)$ is nonsingular, we see that the dual graph of $\sum \psi_{r}^{*}\left(E_{i}\right)$ has no loop.

Case 1. Assume that $E_{0}$ is a component of the branch locus of $\psi_{r}$. In this case, $E_{1}$ is not a component of the branch locus. If $\widetilde{B_{r}}$ intersects $E_{1}$ transversally, then $E_{2}$ is not a component of the branch locus of $\psi_{r}$, because $\psi_{r}^{*}\left(E_{1}\right)$ is a nonsingular rational curve. Moreover, since $\sum_{j=0}^{k} \psi_{r}^{*}\left(E_{j}\right)$ has 
no loop, each $E_{i}(i \geq 2)$ is not a component of the branch locus and does not intersect $\widetilde{B_{r}}$.

Thus, the configuration of $\sum_{j=0}^{k} n_{j} E_{j}$ is as in Figure 1 (a) and $n_{k}=$ $n_{0}, n_{k-1}=2 n_{0}, \ldots, n_{1}=k n_{0}$. In Figure 1 and Figure 2, the dotted lines mean the components of $\sum_{j=0}^{k} n_{j} E_{j}$ which are not components of the branch locus. The solid lines mean the components of the branch locus and the waves mean $\widetilde{B_{r}}$. This contradicts the assumption that all $\psi_{r}^{*}\left(E_{j}\right)_{\text {red }}(j=$ $1,2, \ldots, k)$ are contracted by $\bar{\tau}$. Then, we may assume that $\widetilde{B_{r}}$ does not intersect $E_{1}$ and that $E_{2}$ is a component of the branch locus or $k=1$. When $k=1$, we cannot contract $\psi_{r}^{*}\left(E_{1}\right)$. When $k \neq 1$, by an argument similar to that above, the configuration of $\sum_{j=0}^{k} n_{j} E_{j}$ must be as in Figure 2 (a). Considering an inverse of Horikawa's canonical resolution, i.e., contracting $\sum_{j=1}^{k} n_{j} E_{j}$, we see that $\widetilde{B}_{i}$ intersects $E$ transversally at $Q$, a contradiction.

Case 2. Assume that $E_{0}$ is not a component of the branch locus of $\psi_{r}$. If $E_{1}$ is not a component of the branch locus and $E_{1}$ intersects $\widetilde{B_{r}}$, then the dual graph of $\sum_{j=0}^{k} n_{j} \psi_{r}^{*}\left(E_{j}\right)$ has a loop, a contradiction. Thus, $E_{1}$ does not intersect $\widetilde{B_{r}}$, if we assume that $E_{1}$ is not a component of the branch locus. However, in this case, each $E_{j}(j>1)$ does not intersect $\widetilde{B_{r}}$, because the dual graph of $\sum \psi_{r}^{*}\left(E_{j}\right)$ has no loop. Hence, we see that the configuration of $\sum_{j=0}^{k} n_{j} E_{j}$ must be as in Figure $1(\mathrm{~b})$. This contradicts the assumption that $Q$ is a bad point. Thus, we see that $E_{1}$ is a component of the branch locus. By the same arguments as in Case 1 , the configuration of $\sum_{j=0}^{k} n_{j} E_{j}$ is as in Figure 2 (b), a contradiction to the assumption that $Q$ is a bad point.

When the dual graph of $\sum n_{j} E_{j}$ is not linear, by the assumption that $\sum n_{j} \psi_{r}^{*}\left(E_{j}\right)$ is contracted to a point, we can find the composite of the contractions $\tau^{\prime \prime}$ of $(-1)$-curves such that $\tau^{\prime \prime}\left(\sum n_{j} \psi_{r}^{*}\left(E_{j}\right)\right)$ is linear. Then, we can reduce the arguments to the case where the dual graph of $\sum n_{j} \psi_{r}^{*}\left(E_{j}\right)$ is linear.

COROllary 1.2. In the notation as above, we also assume that $E_{0}$ is a component of the branch locus. If $\bar{\tau}\left(\left(\psi_{r}^{*}\left(E_{0}\right)\right)_{\text {red }}\right)$ is a nonsingular curve and $\bar{\tau}\left(\psi_{r}^{*}\left(\Sigma E_{j}\right)\right)$ is a nonsingular rational curve which transversally intersects $\psi_{r}^{*}\left(E_{0}\right)_{\text {red }}$ at a point, then $E$ intersects $\widetilde{B}_{i}$ at $Q$ transversally.

Proof. By Lemma 1.1, we may assume that the dual graph of $\sum_{j=0}^{k} n_{j} E_{j}$ is linear. Since the dual graph of $\bar{\tau}\left(\psi_{r}^{*}\left(\Sigma E_{j}\right)\right)$ has no loop, by the same argument as in the proof of Lemma 1.1, the configuration of $\sum_{j=0}^{k} n_{j} E_{j}$ must be as in Figure 1 (a) or Figure 2 (a). The configuration is not as in 
Figure 1 (a) by the assumption that $\bar{\tau}\left(\psi_{r}^{*}\left(\Sigma E_{j}\right)\right)$ is a nonsingular rational curve. Hence, we see that the configuration is as in Figure 2 (a) and $k=1$.

Lemma 1.3. In the notation as above, let $(\phi, S, \Delta)$ be a normally minimal hyperelliptic family whose monodromy is periodic. Let $E$ be the exceptional set of $\tau_{i}: W_{i} \longrightarrow W_{i-1}$ such that $\widetilde{\tau}\left(\psi_{r}^{*}\left(E_{0}\right)\right)$ is the main component of $(\phi, S, \Delta)$. If $Q$ is a bad point at which $\widetilde{B}_{i}$ intersects $E$ and if $Q$ is not contained in the exceptional sets of $\tau_{1} \circ \ldots \circ \tau_{i}$ other than $E$, then $\widetilde{B}_{i}$ intersects E transversally at $Q$.

Proof. Let $\sum_{i=1}^{k} n_{i} E_{i}$ be the maximal subdivisor of $\left(\tau_{1} \circ \ldots \circ \tau_{r}\right)^{*}\left(\Gamma_{0}\right)$ which is contracted to $Q$ by $\tau_{i+1} \circ \cdots \circ \tau_{r}$. Note that, by the properties of the configuration of the special fiber and Lemma 1.1, the dual graph of $\sum_{i=1}^{k} n_{i} \psi_{r}^{*}\left(E_{i}\right)$ is linear and all $\psi_{r}^{*}\left(E_{i}\right)$ are nonsingular rational curves. Assume that $E_{0}$ is not a component of the branch locus and that $B_{i}$ intersects $E$ at $Q$ not transversally. Since each $n_{i}$ is greater than or equal to the multiplicity of $E$, the multiplicity of each $\psi_{r}^{*}\left(E_{i}\right)_{\text {red }}$ is greater than or equal to that of the main component. Since $\sum_{i=1}^{k} n_{i} \psi_{r}^{*} E_{i}$ cannot be contracted to a point by Lemma 1.1, there exists a component of the special fiber of $S$ whose multiplicity is greater than or equal to that of the main component, a contradiction to the properties of the configurations of the special fibers of families with periodic monodromies.

Assume that $E_{0}$ is a component of the branch locus. Since the dual graph of $\sum_{i=1}^{k} n_{i} \psi_{r}^{*}\left(E_{i}\right)$ is linear, we see that the configuration of $\sum_{i=1}^{k} n_{i} E_{i}$ must be as in Figure 2 (a). Hence, by Corollary 1.2, we obtain the assertion.

The points which satisfy the same conditions as $Q$ in Lemma 1.3 are called middle points. We use the same notation as in Lemma 1.3.

Lemma 1.4. Let $(\phi, S, \Delta)$ be a normally minimal hyperelliptic family whose monodromy is periodic but not the identity. Then there exists a component $\widetilde{E}$ of $\left(\tau_{1} \circ \cdots \circ \tau_{r}\right)^{*}\left(\Gamma_{0}\right)$ which satisfies the following conditions:

(i) The multiplicity of $\widetilde{E}$ is one.

(ii) $\widetilde{E}$ intersects $\widetilde{B_{r}}$ at at most one point.

(iii) The vertex corresponding to $\widetilde{E}$ in the dual graph of $\left(\tau_{1} \circ \cdots \circ \tau_{r}\right)^{*}\left(\Gamma_{0}\right)$ is a terminal of the graph. 
Proof. Let $M$ be the component of $\left(\tau_{1} \circ \cdots \circ \tau_{r}\right)^{*} \Gamma_{0}$ such that $\widetilde{\tau}\left(\psi_{r}^{*}(M)\right)$ is the main component. By an inverse of Horikawa's canonical resolution, we can consider a composite $\tau_{1} \circ \cdots \circ \tau_{r^{\prime}}$ of blowing-ups such that $\widetilde{B_{r^{\prime}}}$ is nonsingular except at middle points. Since the multiplicity of a terminal component is one, we can find a component $\widetilde{E}$ satisfying (i) and (iii). Since the monodromy is not the identity, $\psi_{r}^{*}(\widetilde{E})$ is a nonsingular rational curve. Thus, $\widetilde{B_{r}}$ intersects $\widetilde{E}$ at at most two points. Suppose $\widetilde{B_{r}}$ intersects $\widetilde{E}$ at two points. Let $M+\widetilde{E}+\Sigma_{i=1} E_{i}$ be the minimal connected subdivisor of $\left(\tau_{1} \circ \cdots \circ \tau_{r}\right)^{*}\left(\Gamma_{0}\right)$ which contains $\widetilde{E}$ and $M$. Then, $\widetilde{E}$ intersects $\Sigma_{i=1} E_{i}$ at a point which is not contained in the branch locus. Thus, the dual graph of $\psi_{r}^{*}\left(M+\widetilde{E}+\Sigma_{i=1} E_{i}\right)$ has at least one loop. This contradicts the assumption that the monodromy is periodic.

TheOREM 1.5. Let $\mathcal{P} \mathcal{H}_{g}$ be the set of the conjugacy classes of periodic maps in the mapping class group of genus $g$ which are realized as the monodromies of hyperelliptic families. Let $(x, t)$ be local coordinates of $\mathbf{P}^{1} \times \Delta$ and $\mathcal{E}_{g}$ the set of double coverings over $\mathbf{P}^{1} \times \Delta$ defined by the following equations:

(I) $y^{2}=(x-1) \Pi_{i=1}^{\delta}\left(x^{p}-\alpha_{i} t^{q}\right)$,

(II) $y^{2}=x(x-1) \Pi_{i=1}^{\delta}\left(x^{p}-\alpha_{i} t^{q}\right)$,

(III) $y^{2}=\Pi_{i=1}^{\delta}\left(x^{p}-\alpha_{i} t^{q}\right)$,

(IV) $y^{2}=t \Pi_{i=1}^{\delta}\left(x^{p}-\alpha_{i} t^{q}\right)$,

(V) $y^{2}=(x-1) \Pi_{i=1}^{2 g+1}\left(x-\alpha_{i} t\right)$,

(VI) $y^{2}=\Pi_{i=1}^{2 g+2}\left(x-\alpha_{i}\right)$,

where $p$ and $q$ are relatively prime positive integers with $p \neq 1$ and $\left\{\alpha_{i}\right\}$ is a set of mutually distinct complex numbers. Let $\Theta: \mathcal{E}_{g} \longrightarrow \mathcal{P H}$ be the map which sends a double covering to its monodromy. Then $\Theta$ is surjective.

Proof. For $[f] \in \mathcal{P H}_{g}$, we choose a normally minimal hyperelliptic family $(\phi, S, \Delta)$ whose monodromy is $[f]$. We consider an inverse of Horikawa's canonical resolution associated to a component $E$ which satisfies the conditions of Lemma 1.4. Putting $\left(\tau_{1}^{\prime} \circ \ldots \circ \tau_{r}^{\prime}\right)_{*}(E)$ to be $\Gamma_{0}$, we use the same notation as in the explanation of Horikawa's canonical resolutions. Since $E$ satisfies the conditions of Lemma 1.4, there exist at most two bad points of $B_{0}$ on $\Gamma_{0}$ and there exists a unique bad point which satisfies $m_{p} \geq 3$. Let $\tau_{1} \circ \cdots \circ \tau_{r^{\prime}}$ be the composite of the blowing-ups which appear in the process of Horikawa's canonical resolution such that $\widetilde{B_{r}}$ is free from bad points except middle points. Since the dual graph 
of $\left(\tau_{1} \circ \ldots \circ \tau_{r^{\prime}}\right)^{*}\left(\Gamma_{0}\right)$ is linear and $\widetilde{B_{r^{\prime}}}$ intersects $\left(\tau_{1} \circ \ldots \circ \tau_{r^{\prime}}\right)^{*}\left(\Gamma_{0}\right)_{\text {red }}$ transversally by Lemmas 1.1 and 1.3, we can deform the local equation of $B_{0}$ near the bad point $P$ to $\Pi_{i=1}^{\delta}\left(x^{p_{i}}-\alpha_{i} t^{q_{i}}\right)=0$ or $t \Pi_{i=1}^{\delta}\left(x^{p_{i}}-\alpha_{i} t^{q_{i}}\right)=0$ $\left(2 g+1 \leq \Sigma_{i} p_{i} \leq 2 g+2\right)$. We may assume the pairs $\left(p_{i}, q_{i}\right)$ to be relatively prime integers. Let $n$ be the period of $f$ and $\bar{n}: \Delta^{\prime} \longrightarrow \Delta$ a morphism of degree $n$ branched at the origin. We also use $t$ as a parameter of $\Delta^{\prime}$. Let $\psi_{0}^{(n)}: S_{0}^{(n)}:=S_{0} \times{ }_{\Delta \times \mathbf{P}^{1}}\left(\Delta^{\prime} \times \mathbf{P}^{1}\right) \longrightarrow \Delta^{\prime} \times \mathbf{P}^{1}$ be the base change of $\psi_{0}$ obtained by $\bar{n}$. Then the local equation of the branch locus $B_{0}^{(n)}$ of $\psi_{0}^{(n)}$ near $\bar{n}^{-1}(p)$ is given by $\Pi_{i=1}^{\delta}\left(x^{p_{i}}-\alpha_{i} t^{n q_{i}}\right)=0$ or $t \Pi_{i=1}^{\delta}\left(x^{p_{i}}-\alpha_{i} t^{n q_{i}}\right)=0$. By the definition of the period of monodromy, the nonsingular model of $S_{0}^{(n)}$ is a smooth family of genus $g$.

We consider the case $n \geq 2$. We first consider the canonical resolution of the branch locus whose equation is $\Pi_{i=1}^{\delta}\left(x^{p_{i}}-\alpha_{i} t^{n q_{i}}\right)=0, \Sigma p_{i}=2 g+2$. Assume that there exists $i$ such that $p_{i}>n q_{i} \geq 2$. Since $\Gamma_{0}$ is the tangent line of the curve $\left\{\left(x^{p_{i}}-\alpha_{i} t^{n q_{i}}\right)=0\right\}$ at $(x, t)=(0,0)$, the strict transform of the curve $\left\{\left(x^{p_{i}}-\alpha_{i} t^{n q_{i}}\right)=0\right\}$ by $\tau_{1} \circ \ldots \circ \tau_{r}$ intersects the components whose multiplicities are greater than or equal to two. Note that the number of the irreducible components of the strict transform of the divisor $\left\{\check{\theta}_{i}:=\left(x^{p_{1}}-\right.\right.$ $\left.\left.\alpha_{1} t^{n q_{1}}\right) \cdots\left(x^{p_{i-1}}-\alpha_{i-1} t^{n q_{i-1}}\right)\left(x^{p_{i+1}}-\alpha_{i+1} t^{n q_{i+1}}\right) \cdots\left(x^{p_{\delta}}-\alpha_{\delta} t^{n p_{\delta}}\right)=0\right\}$ is smaller than or equal to $2 g-1$ because $p_{i}$ is greater than or equal to three. Let $M$ be the component such that $\psi_{r}^{*}(M)$ is a nonsingular curve of genus $g$. Since the multiplicity of $M$ is one, the number of the branch points on $M$ is at most $2 g+1$ even if $\left\{\check{\theta}_{i}=0\right\}$ intersects $M$, a contradiction to our assumption that the genus of $\psi_{r}^{*}(M)$ is $g$. Hence we have $p_{i} \leq n q_{i}$ for all $i$.

Let $E_{r^{\prime \prime}}$ be the exceptional set of the blowing-up $\tau_{r^{\prime \prime}}: W_{r^{\prime \prime}} \longrightarrow W_{r^{\prime \prime}-1}$ such that the strict transform of $E_{r^{\prime \prime}}$ by $\tau_{r^{\prime \prime}+1} \circ \ldots \circ \tau_{r}$ is $M$. If there exists a bad point on $E_{r^{\prime \prime}}$, the special fiber of the nonsingular normally minimal model of $S_{0}$ has at least two components by Lemma 1.1. Thus, there exists no bad point on $E_{r^{\prime \prime}}$, and $\widetilde{B_{r^{\prime \prime}}}(n)$ intersects $E_{r^{\prime \prime}}$ transversally at at least $2 g+1$ distinct points.

When $\widetilde{B_{r^{\prime \prime}}^{(n)}}$ intersects $E_{r^{\prime \prime}}$ at $2 g+1$ points, the local equation of $B_{0}^{(n)}$ at the bad point must be $\left(x-\alpha t^{n q_{\delta}}\right) \Pi_{i=1}^{\delta-1}\left(x^{p_{i}}-\alpha_{i} t^{n q_{i}}\right)=0$ and the strict transform of the curve $\left\{\Pi_{i=1}^{\delta-1}\left(x^{p_{i}}-\alpha_{i} t^{n q_{i}}\right)=0\right\}$ by $\tau_{1} \circ \cdots \circ \tau_{r^{\prime \prime}}$ intersects $E_{r^{\prime \prime}}$ at $2 g+1$ distinct points. Since the multiplicity of $E_{r^{\prime \prime}}$ is one and $p_{i} \leq n q_{i}$, we see that $n q_{i}$ is the multiple of $p_{i}$ and $n q_{i} / p_{i}=n q_{j} / p_{j}$ for all $i, j(1 \leq i, j \leq \delta-1)$. Then, we see that $p_{i}=p_{j}$ and $q_{i}=q_{j}$ because of $\operatorname{gcd}\left(p_{i}, q_{i}\right)=1$ for all $i$. 
When $\widetilde{B_{r^{\prime \prime}}^{(n)}}$ intersects $E_{r^{\prime \prime}}$ at $2 g+2$ points, we can conclude that the local equation of $B_{0}^{(n)}$ is given by $\Pi_{i=1}^{\delta}\left(x^{p}-\alpha_{i} t^{n q}\right)=0$ by similar arguments. From the above arguments, when $\Gamma_{0}$ is not a component of the branch locus of $\psi_{0}$ and $\Sigma p_{i}=2 g+2$, we see that the local equation of $B_{0}$ is given by $\left(x-\alpha t^{q_{\delta}}\right) \Pi_{i=1}^{\delta-1}\left(x^{p}-\alpha_{i} t^{q}\right)=0$ or $\Pi_{i=1}^{\delta}\left(x^{p}-\alpha_{i} t^{q}\right)=0$. If $p>q$, then two complex surfaces of the nonsingular models of $y^{2}=\left(x-\alpha t^{q_{\delta}}\right) \Pi_{i=1}^{\delta-1}\left(x^{p}-\right.$ $\left.\alpha_{i} t^{q}\right)$ and $y^{2}=x \Pi_{i=1}^{\delta-1}\left(x^{p}-\alpha_{i} t^{q}\right)$ have the same monodromy. Let $[q / p]$ be the greatest integer not exceeding $q / p$. If $p<q$, then, by repeating the elementary transformation in the sense of $[\mathrm{Hol}][q / p]+1$ times, we obtain the equation of type (I)

Next we consider the case of $y^{2}=\Pi_{i=1}^{\delta}\left(x^{p_{i}}-\alpha_{i} t^{q_{i}}\right), \Sigma p_{i}=2 g+1$. By arguments similar to those above, we see that $p_{i} \leq n q_{i}$ and that the $n q_{i}$ is a multiple of $p_{i}$. Since, for each $i$, all the irreducible components of the strict transform of $\left\{\left(x^{p_{i}}-\alpha_{i} t^{n q_{i}}\right)=0\right\}$ must intersect $M$ whose multiplicity is one, we conclude that $n q_{i} / p_{i}=n q_{j} / p_{j}$. Hence, we have $q_{i}=q_{j}$ and $p_{i}=p_{j}$ because $\operatorname{gcd}\left(p_{i}, q_{i}\right)=1$. When the local equation of $B_{0}$ at the bad point is $t \Pi_{i=1}^{\delta}\left(x^{p_{i}}-\alpha_{i} t^{q_{i}}\right)=0$, we conclude that $p_{i}=p_{j}$ and $q_{i}=q_{j}$ by similar arguments. Thus, it is sufficient to consider only the following equations as the defining equation of $S_{0}$ :

$$
y^{2}=(x-1) \Pi_{i=1}^{\delta}\left(x^{p}-\alpha_{i} t^{q}\right)
$$

(II) $y^{2}=x(x-1) \Pi_{i=1}^{\delta}\left(x^{p}-\alpha_{i} t^{q}\right)$,

(III) $y^{2}=\Pi_{i=1}^{\delta}\left(x^{p}-\alpha_{i} t^{q}\right)$,

(IV) $y^{2}=t \prod_{i=1}^{\delta}\left(x^{p}-\alpha_{i} t^{q}\right)$,

(V) $y^{2}=(x-1) \Pi_{i=1}^{2 g+1}\left(x-\alpha_{i} t\right)$,

(VI) $y^{2}=\Pi_{i=1}^{2 g+2}\left(x-\alpha_{i}\right)$,

(VII) $y^{2}=t x(x-1) \Pi_{i=1}^{\delta}\left(x^{p}-\alpha_{i} t^{q}\right)$,

(VIII) $y^{2}=t(x-1) \prod_{i=1}^{\delta}\left(x^{p}-\alpha_{i} t^{q}\right)$,

(IX) $y^{2}=x \prod_{i=1}^{\delta}\left(x^{p}-\alpha_{i} t^{q}\right)$,

(X) $y^{2}=t x \Pi_{i=1}^{\delta}\left(x^{p}-\alpha_{i} t^{q}\right)$,

where $p$ and $q$ are relatively prime positive integers with $p \neq 1$ and $\left\{\alpha_{i}\right\}$ is a set of mutually distinct complex numbers. Note that, in the case of the equations (I) through (IV) and (VII) through (X) with $p=1$, we can transform them to the equations of $(\mathrm{V})$ or (VI) by elementary transformations. The equation of $(\mathrm{V})$ gives the very important monodromy, hyperelliptic involution. The equation of (VI) gives the monodromy whose period is one, i.e., the identity which is not covered in the above arguments. However, 
the equations of (VII) through (X) are transformed to (I) by a suitable elementary transformation. In the cases of (VII) and (VIII), the blowing-up at $(x, t)=(1,0)$ and the blowing-down of a $(-1)$-curve which is the strict transform of $\Gamma_{0}$ give the type (I). In the case of (IX), $p>q$, the blowing-up at $(x, t)=(0,0)$ and the blowing-down of a $(-1)$-curve which is the strict transform of $\Gamma_{0}$ give the type (I) or (VIII). We see that (VIII) is reduced to (I). In the case of (IX) $p<q$, we can reduce this to the case of (IX) of $p>q$ by suitable elementary transformations. By similar arguments, we see that the equations of the type of $(\mathrm{X})$ can be transformed to the type (I) by elementary transformations. Hence, we see that $[f]$ is in the image of $\Theta$.

Remark 1.6. Since any hyperelliptic family is realized as the nonsingular model of a double covering of $\mathbf{P}^{1} \times \Delta$, the monodromies of hyperelliptic families are induced by the monodromies of $\mathbf{P}^{1} \times \Delta \backslash B_{0} \longrightarrow \Delta$ defined by the lifting of the vector field of a simple closed curve on $\Delta$, where $B_{0}$ is the branch locus of the double covering. Then it is clear that $\mathcal{P} \mathcal{H}_{g}$ is a subset of $\mathcal{H}_{g}^{p}$, where $\mathcal{H}_{g}^{p}$ is the set of the conjugacy classes of periodic maps which commute with one of the mutually conjugate hyperelliptic involutions in the mapping class group of genus three. Conversely, let $F$ be a periodic map of order $n$ which is an element of one of the mutually conjugate hyperelliptic mapping class groups in the mapping class group. Since the group $G$ generated by $F$ and a hyperelliptic involution is a finite group, by Kerchhoff's theorem (cf. [Ke]), $G$ acts on a hyperelliptic curve $C$ holomorphically. Thus we can regard $F$ as a holomorphic automorphism of $C$. We define the map $\widetilde{F}: C \times \Delta \longrightarrow C \times \Delta$ by $\widetilde{F}(P, t)=\left(F(P), e^{2 \pi i / n} t\right)$. Since $\widetilde{F}$ is holomorphic and the nonsingular model $S_{\widetilde{F}}$ of the quotient space $(C \times \Delta) /\langle\widetilde{F}\rangle$ is a complex surface, $S_{\widetilde{F}}$ is a hyperelliptic family whose monodromy is the conjugacy class of $F$. Hence $\mathcal{H}_{g}^{p}$ is equal to $\mathcal{P} \mathcal{H}_{g}$.

Corollary 1.7. Let $p$ and $q$ be relatively prime integers. A periodic monodromy which is realized as a certain hyperelliptic family of genus $g$ is one of the following types:

(i) $n=2 p ; \sigma_{1} / 2 p+\sigma_{2} / p+\underbrace{1 / 2+\cdots+1 / 2}_{\delta \text { times }}$, where $p \delta=2 g+1, q \sigma_{1} \equiv 1$ $(\bmod 2 p), \lambda(p-1) / 2 \equiv 1 \quad(\bmod p), q \lambda \sigma_{2} \equiv 1 \quad(\bmod p)$ and $q$ is odd.

(ii) $n=p, g^{\prime}=(\delta-1) / 2 ; \sigma_{1} / p+\sigma_{2} / p+\sigma_{2} / p$, where $p \delta=2 g+1$, $q \sigma_{1} \equiv 1, \lambda(p-1) / 2 \equiv 1, \lambda q \sigma_{2} \equiv 1 \quad(\bmod p)$. 
(iii) $n=2 p ; \sigma_{1} / 2 p+\sigma_{2} / 2 p+\underbrace{1 / 2+\cdots+1 / 2}_{\delta \text { times }}$, where $p \delta=2 g, q \sigma_{1} \equiv$ $1, \lambda(2 p-1) \equiv 1, q \lambda \sigma_{2} \equiv 1 \quad(\bmod 2 p), p$ and $\delta$ are even.

(iv) $n=2 p ; \sigma_{1} / 2 p+\sigma_{2} / 2 p+\underbrace{1 / 2+\cdots+1 / 2}_{\delta \text { times }}$, where $p \delta=2 g, q \sigma_{1} \equiv$ $1, \lambda(p-1) \equiv 1, q \lambda \sigma_{2} \equiv 1 \quad(\bmod 2 p), p$ is even and $\delta$ is odd.

(v) $n=2 p ; \sigma_{1} / 2 p+\sigma_{2} / 2 p+\underbrace{1 / 2+\cdots+1 / 2}_{\delta \text { times }}$, where $p \delta=2 g, q \sigma_{1} \equiv$ $1, \lambda(2 p-1) \equiv 1, q \lambda \sigma_{2} \equiv 1 \quad(\bmod 2 p)$, The integers $p$ and $q$ are odd and $\delta$ is even.

(vi) $n=p, g^{\prime}=\delta / 2 ; \sigma_{1} / p+\sigma_{2} / p$, where $p \delta=2 g, q \sigma_{1} \equiv 1, \lambda(p-1) \equiv$ $1, q \lambda \sigma_{2} \equiv 1 \quad(\bmod p), \delta$ is even and $p$ is odd.

(vii) $n=p, g^{\prime}=(\delta-2) / 2 ; \sigma_{1} / p+\sigma_{1} / p+\sigma_{2} / p+\sigma_{2} / p$, where $p \delta=2 g+2$, $q \sigma_{1} \equiv 1, \lambda(p-1) \equiv 1, q \lambda \sigma_{2} \equiv 1, \quad(\bmod p), p$ and $\delta$ are even.

(viii) $n=p, g^{\prime}=(\delta-1) / 2 ; \sigma_{1} / p+\sigma_{1} / p+\sigma_{2} /(p / 2)$, where $p \delta=2 g+2$, $q \sigma_{1} \equiv 1 \quad(\bmod p), \lambda(p-2) / 2 \equiv 1, q \lambda \sigma_{2} \equiv 1 \quad(\bmod p / 2), p$ is even and $\delta$ is odd.

(ix) $n=p, g^{\prime}=(\delta-2) / 2 ; \sigma_{1} / p+\sigma_{1} / p+\sigma_{2} / p+\sigma_{2} / p$, where $p \delta=2 g+2$, $q \sigma_{1} \equiv 1, \lambda(p-1) \equiv 1, q \lambda \sigma_{2} \equiv 1 \quad(\bmod p), p$ is odd and $\delta$ is even.

(x) $n=p, g^{\prime}=\delta / 2 ; \sigma_{1} /(p / 2)+\sigma_{2} /(p / 2)$, where $p \delta=2 g+2, q \sigma_{1} \equiv$ $1, \lambda(p-2) / 2 \equiv 1, q \lambda \sigma_{2} \equiv 1 \quad(\bmod p / 2), p$ and $\delta$ are even.

(xi) $n=2 p ; \sigma_{1} / p+\sigma_{2} / p+\underbrace{1 / 2+\cdots+1 / 2}_{\delta \text { times }}$, where $p \delta=2 g+2, q \sigma_{1}(p+$ $1) / 2 \equiv 1, \lambda(p-1) / 2 \equiv 1, q \lambda \sigma_{2} \equiv 1 \quad(\bmod p), q$ and $p$ are odd, and $\delta$ is even.

(xii) $n=2 ; \underbrace{1 / 2+1 / 2+\cdots+1 / 2}_{2 g+2 \text { times }}$,

where $n$ and the sums of fractional numbers mean the period and the total valency, respectively. $g^{\prime}$ means the genus of the main component but we omit it when $g^{\prime}=0 . \quad p$ and $q$ are relatively prime and $\sigma_{1}, \sigma_{2}, \lambda$ are the smallest positive integers which satisfy each specified condition.

Proof. Consider the case where the equation of $S_{0}$ is (I), $q=1$. By Horikawa's canonical resolution, we see that the configuration of the special fiber of the normally minimal nonsingular model of $S_{0}$ is as in Figure 3 . In Figure 3, the lines mean nonsingular rational curves and the numbers 
beside them are their multiplicities. Thus, the period of the monodromy is $2 p$ and the total valency is $1 / 2 p+(p-1) / 2 p+\underbrace{1 / 2+\cdots+1 / 2}_{\delta \text { times }}$. Taking a suitable representative $f$ of this monodromy, there exists the set of points $\left\{P_{1}, P_{2}, P_{3}, Q_{j}^{i}\right\}(1 \leq i \leq \delta, 1 \leq j \leq p)$ such that $f\left(P_{1}\right)=P_{1}, f^{2}\left(P_{i}\right)=P_{i}$ $(i=2,3)$ and $f^{p}\left(Q_{j}^{i}\right)=Q_{j}^{i}$. Moreover, $f$ is the rotation of angle $2 \pi / 2 p$ near $P_{1}$ and $f^{2}$ is the rotation of angle $2 \pi \lambda / p$ near $P_{2}$ and $P_{3}$, where $\lambda$ satisfies $\lambda(p-1) / 2 \equiv 1 \quad(\bmod p) . f^{p}$ is the rotaion of angle $\pi$ near $Q_{j}^{i}$. By a base change of degree $q$ such that $q$ is odd and $\operatorname{gcd}(p, q)=1$, we can make a hyperelliptic family with monodromy $\left[f^{q}\right]$. The map $f^{q}$ is the rotation of angle $2 \pi q / 2 p$ near $P_{1},\left(f^{q}\right)^{2}$ is the rotation of angle $2 \pi \lambda q / p$ near $P_{2}$ and $P_{3}$, and $f^{p}$ is the rotaion of angle $\pi$ near $Q_{j}^{i}$. Then we obtain the monodromy of type (i).

By arguments similar to that above, the monodromies (ii) are obtained by suitable base changes of the equation of type (I), $q=2$. The monodromies (iii),(iv) and (v) are obtained from the case of the equation of type (II), $q=1$. The monodromies (vi) are obtained from the case of type (II), $q=2$. The monodromies (vii),(viii) and (ix) are obtained from the case of the equation of type (III), $q=1$. The monodromies (x) and (xi) are obtained from the case of the equation of type (IV), $q=1$. Note that the monodromies which are obtained from the cases of the equations of type (IV) and $q$ even are equal to (xi). The monodromy (xii) is obtained from the case of the equation of type $(\mathrm{V})$.

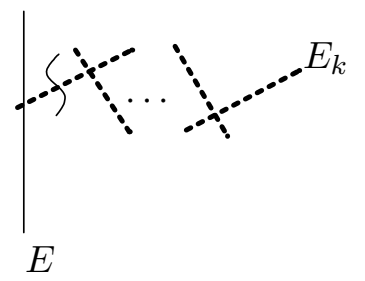

(a)

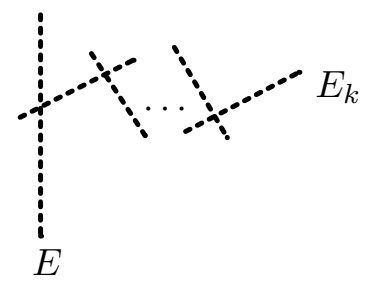

(b)

Figure 1 


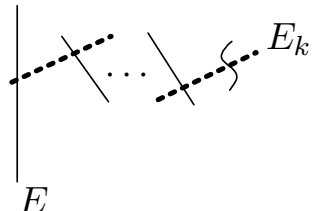

(a)

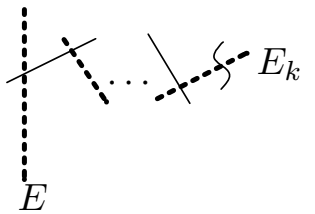

(b)

Figure 2

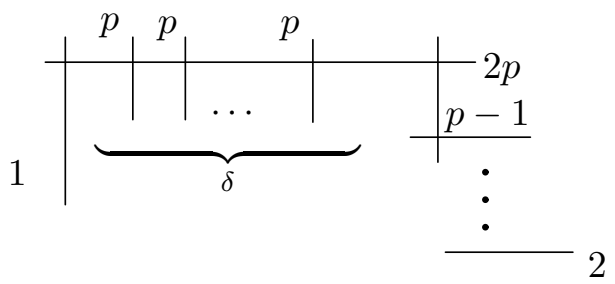

Figure 3

\title{
REFERENCES
}

[AA] T. Arakawa and T. Ashikaga, Local splitting families of hyperelliptic pencils I, Tohoku Math. J., 53 (2001), 369-394.

[AI] T. Ashikaga and M. Ishizaka, Classification of degenerations of curves of genus three via Matsumoto-Montesinos' theorem, Tohoku Math. J., 54 (2002), 195-226.

[Ho1] E. Horikawa, On deformations of quintic surfaces, Invent. Math., 31 (1975), 43-85.

[Ho2] - On algebraic surfaces with pencils of curves of genus 2, in Complex Analysis and Algebraic Geometry, a collection of papers dedicated to K. Kodaira, pp.79-90, Iwanami Shoten, Tokyo and Cambridge Univ. Press, 1977.

[Ke] S. P. Kerchhoff, The Nielsen realization problem, Ann. of Math., 117 (1983), 235-265.

[MM1] Y. Matsumoto and J. M. Montesinos-Amilibia, Pseudo-periodic maps and degeneration of Riemann surfaces I, II, preprints, Univ. of Tokyo and Univ. Complutense de Madrid, 1991/1992.

[MM2] _ Pseudo-periodic homeomorphisms and degeneration of Riemann surfaces, Bull. Amer. Math. Soc., 30 (1994), 70-75.

\author{
Mathematical Institute \\ Tohoku University \\ Sendai 980-8578 \\ Japan \\ ishizakamizuho@aoni.waseda.jp
}

